\title{
Small instantons and the strong $C P$ problem in composite Higgs models
}

\author{
R. S. Gupta, V. V. Khoze, and M. Spannowsky \\ Institute for Particle Physics Phenomenology, Durham University, \\ South Road, Durham DH1 3LE, United Kingdom
}

(Received 24 March 2021; accepted 9 September 2021; published 7 October 2021)

\begin{abstract}
We show that QCD instantons can generate large effects at small length scales in the ultraviolet in standard composite Higgs models that utilize partial compositeness. This has important implications for possible solutions of the strong $C P$ problem in these models. First we show that in the simplest known UV completions of composite Higgs models, if an axion is also present, it can have a mass much larger than the usual QCD axion. Even more remarkable is the case where there are no axions, but the strong $C P$ problem can be solved by generating the up quark mass entirely from the contribution of instantons thus reviving the massless up-quark solution for these models. In both cases no additional field content is required apart from what is required to realize partial compositeness.
\end{abstract}

DOI: 10.1103/PhysRevD.104.075011

\section{INTRODUCTION}

The strong $C P$ problem is one of the five major particle physics puzzles that motivate the existence of new physics beyond the Standard Model (SM). The most elegant solution to this problem is the existence of a new $U(1)$ symmetry that is anomalous under QCD so that it is possible to rotate away the strong $C P$ phase. The simplest possibility is that the up quark is massless which leads to the existence of an axial $U(1)$ symmetry that makes the strong $C P$ phase unphysical. Another possibility is the existence of the Peccei-Quinn $U(1)$ symmetry that is spontaneously broken resulting in a Goldstone mode, the axion. As the Peccei-Quinn symmetry is anomalous under QCD, the axion gets a potential due to nonperturbative QCD effects and stabilizes at a value that leads to a vanishing strong $C P$ phase [1-3].

Both the above solutions are thought to have unambiguous low energy consequences. The massless up quark solution [4-8] can be tested by lattice simulations. Unfortunately the latest lattice studies indicate a nonzero up mass, that seemingly falsifies this possibility $[9,10]$. This leaves the Peccei-Quinn solution which predicts the existence of the axion with a mass and coupling that is restricted to lie in a narrow band in the parameter space. A vigorous experimental effort that aims to probe the full band is currently underway.

Published by the American Physical Society under the terms of the Creative Commons Attribution 4.0 International license. Further distribution of this work must maintain attribution to the author(s) and the published article's title, journal citation, and DOI. Funded by SCOAP ${ }^{3}$.
The above predictions, however, rely on the tacit assumption that any nonperturbative contribution to the up mass in the first case or to the axion in the second case, arises from the large instantons in the IR. If small instantons in the UV also become important it will completely alter the above experimental expectations. Previous attempts to enhance these UV contributions to the axion mass require additional elements-such as new colored fermions [11,12], extra dimensions [13] or a UV modification of the QCD gauge group [14-16].

In this work we show that small instanton contributions can become important in composite Higgs models with partially composite fermions $[17,18]$. This can be achieved with no additional field content other than what is necessary to fully realize partial compositeness in standard UV completions of these models.

The enhancement of small instanton effects in composite model is possible because the two factors that suppress small instanton contributions in the SM, namely, the smallness of the strong coupling in the UV and the smallness of the product of the SM Yukawa couplings, can both be overcome in these models. The first suppression factor can be overcome because, as we will show, in order to generate composite partners for all SM fermions, many new colored fermions need to be introduced. These new degrees of freedom alter the running of the QCD strong coupling in the UV where it grows again to nonperturbative values. As far as the suppression due to the Yukawa couplings is concerned, this can be overcome because in these models the effective SM Yukawa matrices can be anarchic and $\mathcal{O}(1)$ in the UV.

We show that the enhancement of the small instanton contributions can be so effective in these models that it may be possible to generate the entire mass of the up quark from 
TABLE I. The two-component left-handed fermions of the UV theory. The confining hypercolor gauge group is $G_{\mathrm{HC}}$ and $G_{\mathrm{F}}=$ $S U(5) \times S U(3) \times S U(3)^{\prime} \times S U(3)_{F}^{4} \times U(1)_{X} \times U(1)_{B} \times U(1)_{A 1} \times U(1)_{A 2}$ is the global symmetry group before symmetry breaking where $S U(3)_{F}^{4}=S U(3)_{F}^{u 1} \times S U(3)_{F}^{u 2} \times S U(3)_{F}^{d 1} \times S U(3)_{F}^{d 2}$.

\begin{tabular}{cccccccccc}
\hline \hline & $S U(4)_{\mathrm{HC}}$ & $S U(5)$ & $S U(3)$ & $S U(3)^{\prime}$ & $S U(3)_{F}^{4}$ & $U(1)_{X}$ & $U(1)_{B}$ & $U(1)_{A 1}$ & $U(1)_{A 2}$ \\
\hline$\psi$ & $\mathbf{6}$ & $\mathbf{5}$ & $\mathbf{1}$ & $\mathbf{1}$ & $(\mathbf{1}, \mathbf{1}, \mathbf{1}, \mathbf{1})$ & 0 & 0 & $-18 / 5$ & 0 \\
$\chi_{u}^{i}$ & $\mathbf{4}$ & $\mathbf{1}$ & $\mathbf{3}$ & $\mathbf{1}$ & $(\mathbf{3}, \mathbf{1}, \mathbf{1}, \mathbf{1})$ & $-1 / 3$ & $-1 / 6$ & 1 & 1 \\
$\tilde{\chi}_{u}^{i}$ & $\overline{\mathbf{4}}$ & $\mathbf{1}$ & $\mathbf{1}$ & $\overline{\mathbf{3}}$ & $(\mathbf{1}, \overline{\mathbf{3}}, \mathbf{1}, \mathbf{1})$ & $1 / 3$ & $1 / 6$ & 1 & 1 \\
$\chi_{d}^{j}$ & $\mathbf{4}$ & $\mathbf{1}$ & $\mathbf{3}$ & $\mathbf{1}$ & $(\mathbf{1}, \mathbf{1}, \mathbf{3}, \mathbf{1})$ & $1 / 6$ & $-1 / 6$ & 1 & -1 \\
$\tilde{\chi}_{d}^{j}$ & $\overline{\mathbf{4}}$ & $\mathbf{1}$ & $\mathbf{1}$ & $\overline{\mathbf{3}}$ & $(\mathbf{1}, \mathbf{1}, \mathbf{1}, \overline{\mathbf{3}})$ & $-1 / 6$ & $1 / 6$ & 1 & -1 \\
\hline \hline
\end{tabular}

instanton effects. This leads to a solution of the strong $C P$ problem as in the deep UV the up Yukawa is absent and indeed an additional $U(1)$ symmetry related to the axial rotation of left and right handed up quarks exists; such a chiral rotation can be used to completely rotate away the strong $C P$ phase. We also show that in an alternative scenario where an axion field exists, its mass would lie outside the usual band for the QCD axion because of the enhancement of strong instanton effects in these models.

\section{MODEL}

We consider a straightforward extension of one of the simplest known UV completions of composite models $[17,18]$ by Ferretti [19] where the confining hypercolor gauge group is $S U(4)_{\mathrm{HC}}$. The field content of our model is shown in Table I. The original model in Ref. [19] only has a single pair of fermions, $\chi_{u}$ and $\tilde{\chi}_{u}$, and it can generate partners only for one left handed doublet and one right handed up-type quark. In order to obtain partners for all SM fermions we have extended the field content of the original model by simply taking three copies, $\chi_{u}^{i}$ and $\tilde{\chi}_{u}^{i}$ with $i=1-3$, and also introducing the analogous fields $\chi_{d}^{j}$ and $\tilde{\chi}_{d}^{j}$ that will give rise to partners for the down-type SM fermions The Lagrangian,

$\mathcal{L}_{k}=-i\left(\bar{\chi}_{u}^{i} \not D \chi_{u}^{i}+\bar{\chi}_{d}^{j} \not D \chi_{d}^{j}+\overline{\tilde{\chi}}_{u}^{k} \not D \tilde{\chi}_{u}^{k}+\overline{\tilde{\chi}}_{d}^{l} \not D \tilde{\chi}_{l}^{j}+\bar{\psi} \not D \psi\right)$

thus has an additional $S U(3)_{F}^{4}=S U(3)_{F}^{u 1} \times S U(3)_{F}^{u 2} \times$ $S U(3)_{F}^{d 1} \times S U(3)_{F}^{d 2}$ symmetry not present in the model in Ref. [19]. Note that in our notation above we have only made the index, $\{i, j, k, l\}$, corresponding to the $S U(3)_{F}^{4}$ symmetry explicit.

\section{A. Running of hypercolor coupling}

With the additional matter content, the hypercolor group in our model is no longer asymptotically free as in the original model in Ref. [19]. As is standard for models employing fermionic partial compositeness [17,18], we assume instead, that the theory has a strongly coupled UV fixed point. This is possible, for instance, if the $\beta$-function of the hypercolor gauge coupling has the kind of dependance on the gauge coupling proposed in Ref. [20] and shown in Fig. 1. We will assume that our model lives in the region $g>g_{\star}$ and flows from the UV fixed point $g=g_{\star}$ to larger values $g>g_{\star}$ in the IR where it confines.

\section{B. Global symmetry breaking pattern}

When the hypercolor group $S U(4)_{\mathrm{HC}}$ confines $\psi, \chi_{u, d}^{i}$ and $\tilde{\chi}_{u, d}^{i}$ form TeV-scale condensates,

$$
\begin{aligned}
\left\langle\psi^{p} \psi^{q}\right\rangle & \sim \delta^{p q} f^{d_{\psi \psi \mu}}, \quad\left\langle\chi_{u}^{p, i} \tilde{\chi}_{u}^{p, j}\right\rangle \sim \delta^{i j} f_{\chi_{u}^{\chi_{u} \tilde{\chi}_{u}}}^{d \widetilde{d}}, \\
\left\langle\chi_{d}^{p, i} \tilde{\chi}_{d}^{p, j}\right\rangle & \sim \delta^{i j} f_{\chi_{d}^{\chi_{d} \chi_{d}}}
\end{aligned}
$$

thus breaking the original global symmetry. Here $p, q$ are the indices under the $S U(5)$ global symmetry and $\{i, j\}$ the indices under the $S U(3)_{F}^{u 1, d 1} \times S U(3)_{F}^{u 2, d 2}$ flavor symmetry whereas $d_{\psi \psi}, d_{\chi_{u} \widetilde{\chi_{u}}}$ and $d_{\chi_{d} \widetilde{\chi_{d}}}$ denote the scaling dimensions of the respective condensates. The condensates break $S U(5)$ to $S O(5)$ and $S U(3) \times S U(3)^{\prime}$ down to the diagonal $S U(3)_{c}$ as in Ref. [19]. The coset space, $G_{\mathrm{F}} / H_{\mathrm{F}}$, is given by,

$$
\frac{S U(5) \times S U(3) \times S U(3)^{\prime} \times S U(3)_{F}^{4} \times U(1)^{4}}{S O(5) \times S U(3)_{c} \times S U(3)_{F}^{2} \times U(1)_{X} \times U(1)_{B}}
$$

where $U(1)^{4}=U(1)_{X} \times U(1)_{B} \times U(1)_{A 1} \times U(1)_{A 2}$ and the symmetry $S U(3)_{F}^{4}=S U(3)_{F}^{u 1} \times S U(3)_{F}^{u 2} \times$ $S U(3)_{F}^{d 1} \times S U(3)_{F}^{d 2}$ breaks to the diagonal subgroup $S U(3)_{F}^{2}=S U(3)_{F}^{u} \times S U(3)_{F}^{d}$.

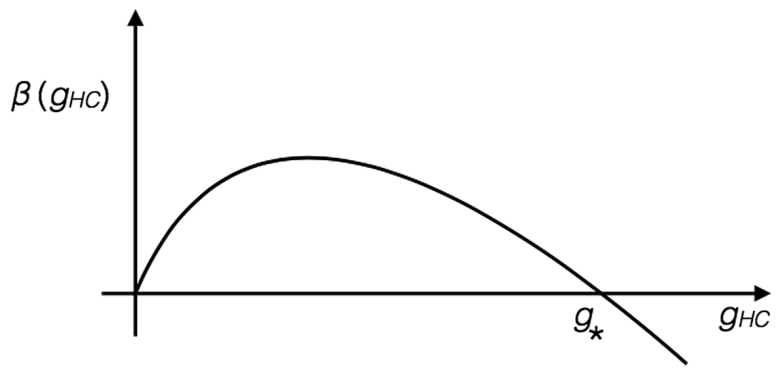

FIG. 1. The assumed dependance of the hypercolor $\beta$-function on the gauge coupling. Our model lives in the region $g>g_{\star}$. 
The unbroken diagonal $S U(3)_{c}$ is gauged to give the QCD Lagrangian. The electroweak group is also gauged. It is embedded in $S O(5)$ as follows,

$$
\begin{aligned}
& S O(5) \times S U(3)_{c} \times U(1)_{X} \\
& \quad \supset G_{\text {cus. }} \equiv S U(3)_{c} \times S U(2)_{L} \times S U(2)_{R} \times U(1)_{X} \\
& \quad \supset G_{\mathrm{SM}} \equiv S U(3)_{c} \times S U(2)_{L} \times U(1)_{Y} \\
& \quad \supset S U(3)_{c} \times U(1)_{\mathrm{e} . \mathrm{m} .},
\end{aligned}
$$

where hypercharge generator is given by $Y=T_{3 R}+X, T_{3 R}$ being the diagonal $S U(2)_{R}$ generator.

The Lagrangian in Eq. (1) is invariant under 5 independent $U(1)$ symmetries, one associated with the rotation of each of the five fermion species $\chi_{u}^{i}, \chi_{d}^{i}, \tilde{\chi}_{u}^{i}, \tilde{\chi}_{u}^{i}$, and $\psi$. Two of these $U(1) \mathrm{s}$ are not spontaneously broken by the condensates in Eq. (2). The first is the linear combinations of these five symmetries that corresponds to the $U(1)_{X}$ symmetry in Table I. The second is a linear combination of these $U(1) \mathrm{s}$ that can be identified with, $U(1)_{B}$, the extension of the baryon number symmetry that includes the new fields. There are three remaining $U(1) \mathrm{s}$ that get broken spontaneously by the condensates. One linear combination of these three $U(1) \mathrm{s}$ is anomalous and thus not a true symmetry, which is why it does not appear in Eq. (3). This still leaves two $U(1)$ s, namely $U(1)_{A 1}$ and $U(1)_{A 2}$, shown in Table I.

There are two Goldstone bosons, $\eta_{1}^{\prime}$ and $\eta_{2}^{\prime}$, corresponding to the spontaneous breaking of $U(1)_{A 1}$ and $U(1)_{A 2}$, respectively. In the electroweak sector the symmetry breaking pattern, $S U(5) \rightarrow S O(5)$ gives rise to 14 pseudoGoldstone bosons. These include the Higgs doublet, $H$, a real singlet, a hyprcharge neutral $S U(2)_{L}$ triplet and a complex $S U(2)_{L}$ triplet charged under hypercharge. As we will discuss shortly, these and all other Goldstone modes become massive once we introduce other terms in the Lagrangian that explicitly break the original global symmetry, $G_{F}$.

\section{Partial compositeness}

We will now discuss how partial compositeness can be realized in this model. We need a composite fermionic partner for each of the SM fermions. In the UV near the fixed point $g=g_{\star}$ in Fig. 1 we identify the following baryonic operators,

$$
\begin{array}{ll}
\mathcal{O}_{u L}^{c, i}=\left(\chi_{u} \psi \chi_{u}\right)^{i} & \mathcal{O}_{u R}^{i}=\left(\bar{\chi}_{u} \bar{\psi} \tilde{\chi}_{u}\right)^{i} \\
\mathcal{O}_{d L}^{c, i}=\left(\chi_{d} \psi \chi_{d}\right)^{i} & \mathcal{O}_{d R}^{i}=\left(\bar{\chi}_{d} \bar{\psi} \tilde{\chi}_{d}\right)^{i},
\end{array}
$$

which have components that have the right transformation properties to be partners of right-handed up type quarks, left-handed up type quarks, right-handed down type quarks and left-handed down type quarks respectively These are all left-handed two component spinor states that transform as triplets under $S U(3)_{c}$. Here $i$ denotes the index corresponding to the $S U(3)_{F}^{u(d)}$ flavor group under which the up type (down type) states transform as a triplet.

The operator $\mathcal{O}_{u R}^{i}$ transforms as $(\mathbf{5}, \mathbf{3})_{2 / 3}$ under $S O(5) \times S U(3)_{c} \times U(1)_{X}$. It has components, that we call $U_{R}^{i}$, which transform as $(\mathbf{3}, \mathbf{1})_{2 / 3}$ under $S U(3)_{c} \times$ $S U(2)_{L} \times U(1)_{Y}$ that can be identified as the partner for $u_{R}^{c, i}$, the antiparticle for the SM right-handed up quarks. Similarly $\mathcal{O}_{u L}^{c, i}$ transforms as $(\mathbf{5}, \overline{\mathbf{3}})_{-2 / 3}$ under $S O(5) \times$ $S U(3)_{c} \times U(1)_{X}$ and has components that transform as $(\overline{\mathbf{3}}, \mathbf{2})_{-2 / 3}$ under $S U(3)_{c} \times S U(2)_{L} \times U(1)_{Y}$. We will call these components, $U_{L}^{c, i}$, and they will serve as partners for the SM left handed up-type fermions.

As far as, $\mathcal{O}_{d R}^{j}$ and $\mathcal{O}_{d L}^{c, j}$, are concerned they transform respectively as $(\mathbf{5}, \mathbf{3})_{-1 / 3}$ and $(\mathbf{5}, \overline{\mathbf{3}})_{1 / 3}$ under $S O(5) \times$ $S U(3)_{c} \times U(1)_{X}$. They, have components that transform respectively as $(\mathbf{3}, \mathbf{1})_{-1 / 3}$ and $(\overline{\mathbf{3}}, \mathbf{2})_{1 / 3}$ under $S U(3)_{c} \times$ $S U(2)_{L} \times U(1)_{Y}$; we will call these $D_{R}^{j}$ and $D_{L}^{c, j}$, the partners for the right-handed antidown quarks and the left-handed down quarks.

The partial compositeness Lagrangian can now be realized by linearly coupling the SM fermions to their partners,

$$
\begin{aligned}
\mathcal{L}_{\text {mix }}= & \frac{\lambda_{u_{R}}^{i j}}{4 \pi} \frac{1}{\Lambda^{d_{U_{R}}-5 / 2}} u_{R}^{c, i} U_{R}^{j}+\frac{\lambda_{u L}^{i j}}{4 \pi} \frac{1}{\Lambda^{d_{U_{L}}-5 / 2}} q_{L}^{i} U_{L}^{c, j} \\
& +\frac{\lambda_{d_{R}}^{i j}}{4 \pi} \frac{1}{\Lambda^{d_{D_{R}}-5 / 2}} d_{R}^{c, i} D_{R}^{j}+\frac{\lambda_{d L}^{i j}}{4 \pi} \frac{1}{\Lambda^{d_{D_{L}}-5 / 2}} q_{L}^{i} D_{L}^{c, j} \\
& + \text { H.c. }
\end{aligned}
$$

where $d_{F}$ is the conformal dimension of the corresponding operator, $F$. The conformal dimensions are independent of the flavor indices $i, j$ because of the $S U(3)_{F}^{u} \times S U(3)_{F}^{d}$ symmetry. The coupling of the SM fermions to the other possible baryonic operators - such as the right-handed SM quarks with $\left(\overline{\tilde{\chi}}_{u, d} \psi \overline{\tilde{\chi}}_{u, d}\right)^{i}$ and $\left(\chi_{u, d} \psi \chi_{u, d}\right)^{i}$ or the left-handed quarks with $\left(\overline{\tilde{\chi}}_{u, d} \psi \overline{\tilde{\chi}}_{u, d}\right)^{i}$ and $\left(\overline{\tilde{\chi}}_{u, d} \bar{\psi} \chi_{u, d}\right)^{i}$-is prohibited as we impose a $Z_{2}$ symmetry under which $\chi_{u, d}^{i}$ and the SM right-handed fermions are odd.

In the IR after confinement the above operators lead to composite states that pair with charge conjugates states (that can be obtained by interchanging $\chi_{u, d} \leftrightarrow \tilde{\chi}_{u, d}$ ) to form massive Dirac fermions. Once these are integrated out the SM Yukawa coupling between the SM fermions and the composite Higgs boson is generated (see, e.g., Ref. [18]).

\section{Explicit breaking by masses and four- $\psi$ interaction}

Finally we add some additional terms not present in the original model of Ref. [19], 
$\mathcal{L}_{\text {new }}=m_{0} e^{i \theta_{m}} \psi_{0} \psi_{0}+\frac{g_{4 \psi} e^{i \theta_{g}}}{\Lambda^{2}}\left(\bar{\psi}_{-+} \bar{\sigma}_{\mu} \psi_{0}\right)\left(\bar{\psi}_{+-} \bar{\sigma}^{\mu} \psi_{0}\right)$

where $m_{0}$ and $g_{4 \psi}$ are real, and the subscript $\{\alpha, \beta\}$ in $\psi_{\alpha \beta}$ in the last term refers to the $T_{3 R, 3 L}$ charges. To make our notation clear and to understand how each of these components of $\psi$ transform, recall that fermion $\psi$ transforms as a 5 of $S U(5)$. This decomposes into two $S U(2)_{L}$ doublets and an $S U(2)_{L}$ singlet,

$$
\left(\begin{array}{c}
\Psi_{+} \\
\Psi_{-} \\
\psi_{0}
\end{array}\right),
$$

$\Psi_{ \pm}$are $\mathrm{SU}(2)_{L}$ doublets and the \pm subscripts correspond to $T_{3 R}= \pm 1 / 2$. We can explicitly write $\Psi_{ \pm}^{T}=\left(\psi_{ \pm+}, \psi_{ \pm-}\right)$ where the second index now corresponds to the $T_{3 L}= \pm 1 / 2$.

The first term breaks $U(1)_{A 1}$ and thus gives a mass to $\eta_{1}^{\prime}$. The $\eta_{1}^{\prime}$ does not get a contribution form the mixing terms in Eq. (6) as we can extend the $U(1)_{A 1}$ symmetry to the SM fermions in a way that is preserved by Eq. (6), i.e., by giving the $U(1)_{A 1}$ charges, $8 / 5$ and $18 / 5$, respectively to the SM doublet and singlet fermions. The $\eta_{1}^{\prime}$ actually would eventually get a contribution to its mass also from nonperturbative QCD effects as the above extended $U(1)_{A 1}$ is anomalous under QCD. On the other hand, $U(1)_{A 2}$ is already broken by the mixing terms in Eq. (6) which give $\eta_{2}^{\prime}$ a mass.

The second term in Eq. (7) is a four- $\psi$ interaction between components of the fermion $\psi$ that explicitly breaks the original global symmetry $S U(5)$. This term would be essential in enhancing the QCD small instanton contributions in the next section.

\section{E. A minimally flavor and $\boldsymbol{C P}$ violating strong sector}

Notice that or model defined by lagrangian in Eq. (1) is invariant under $C P$ and the $S U(3)_{F}^{u} \times S U(3)_{F}^{d}$ flavor symmetry even after the condensates in Eq. (2) are formed. These symmetries are broken only by the couplings $\lambda_{u L}^{i j}, \lambda_{u R}^{i j}, \lambda_{d L}^{i j}, \lambda_{d R}^{i j}, g_{4 \psi} e^{i \theta_{g}}, m_{\phi} e^{i \theta_{m}}$ and the strong $C P$ phases in the QCD and hypercolor sectors, $\theta_{\mathrm{QCD}}$ and $\theta^{\prime}$ respectively. Following Ref. [21], here we will further assume that the mixings of the right handed quarks,

$$
\lambda_{u R, d R}^{i j} \sim y_{u R, d R} e^{i \theta_{R}} \delta^{i j},
$$

do not break the $S U(3)_{F}^{u} \times S U(3)_{F}^{d}$ symmetry when appropriately extended to include the three generations of SM right handed quarks as triplets. This implies that the SM Yukawa couplings would be proportional to the lefthanded mixings,

$$
\begin{gathered}
Y_{u}^{i j} \sim \frac{\lambda_{u L}^{i k} \lambda_{u R}^{k j}}{4 \pi} \sim \lambda_{u L}^{i j} y_{u R} \\
Y_{d}^{i j} \sim \frac{\lambda_{d L}^{i k} \lambda_{d R}^{k j}}{4 \pi} \sim \lambda_{d L}^{i j} y_{d R} .
\end{gathered}
$$

Note that in the limit $\lambda_{u L, d L}^{i j} \rightarrow 0$ the full $U(3)^{3}$ SM flavor symmetry is recovered. Our, setup thus realizes minimal flavor violation (MFV) [22] with the couplings $\lambda_{u L, d L}^{i j}$-that transform like the SM Yukawa terms-being the only spurions that break the $U(3)^{3}$ flavor symmetry.

As far as $C P$ phases, $\theta_{m}, \theta_{g}, \theta_{R}, \theta_{\mathrm{QCD}}$, and $\theta^{\prime}$ are concerned, we can transfer all of them to $\lambda_{u L}^{i j}$ and $\lambda_{d L}^{i j}$ by taking the following steps:

(1) First, the phase $\theta_{m}$ can be rotated away by $\psi_{0} \rightarrow$ $\psi_{0} e^{-i \theta_{m} / 2}$ which redefines $\theta_{g}, \theta_{R}$ and $\theta^{\prime}$.

(2) Next, the phase $\theta_{g}$ associated to $g_{4 \psi}$ can be rotated to $\lambda_{u L}^{i j}$ and $\lambda_{d L}^{i j}$ by making the transformation $\psi_{-+} \rightarrow \psi_{-+} e^{i \theta_{g}}$. This also redefines $\theta^{\prime}$.

(3) Then $\theta^{\prime}$ can be eliminated by an equal rotation of all $\chi_{i}$ and $\tilde{\chi}_{i}$, which also redefines $\theta_{\mathrm{QCD}}$.

(4) Finally $\theta_{R}$ can be eliminated by an equal but opposite rotation of the $\chi_{i}$ relative to the $\tilde{\chi}_{i}$.

This still leaves $\theta_{\mathrm{QCD}}$ which can be entirely shifted to $\lambda_{u L}^{i j}$ and $\lambda_{d L}^{i j}$ by chiral rotations of the SM quarks while keeping the combination,

$$
\bar{\theta}_{\mathrm{QCD}}=\theta_{\mathrm{QCD}}+\operatorname{ArgDet}\left[\lambda_{u} \lambda_{d}\right]
$$

unchanged. Because we have a MFV like structure, as in the SM, there is only one more physical phase in our theory, the CKM phase,

$$
\theta_{\mathrm{CKM}}=\operatorname{ArgDet}\left[\lambda_{u} \lambda_{d}-\lambda_{d} \lambda_{u}\right] .
$$

\section{EFFECT OF SMALL INSTANTONS}

The effect of QCD instantons at high energies are suppressed due to two reasons, (1) the suppression factor $\kappa_{s}=e^{-2 \pi / \alpha_{s}}$ is small as QCD is asymptotically free, and, (2) there is a suppression factor that goes as the product of the Yukawa couplings of all the SM quarks, all of which are active at high energies. Both these effects can be overcome in the model we are considering because, (1) the new colored fermions $\chi_{u, d}^{i}$ and $\tilde{\chi}_{u, d}^{i}$ that form the composite fermionic partners can lead to large UV values of the QCD coupling and (2) the mixings, $\lambda_{u L, d L}^{i j}$, and thus the Yukawas in Eq. (10) can run to higher values in the UV in these models as we will now show.

\section{A. Running of $\boldsymbol{\alpha}_{s}$}

In our model there are 8 new flavors of fermions for every generation once we take into account the 4 hypercolor 
degrees of freedom of $\chi_{i}^{u, d}, \tilde{\chi}_{i}^{u, d}$. Including the SM fermions, there are $n_{f}=30$ flavors. Using the usual expression,

$$
\frac{d g_{s}}{d \log \mu}=-\left(11-2 n_{f} / 3\right) \frac{g_{s}^{3}}{16 \pi^{2}}
$$

we find that the QCD beta function is positive. Assuming that the new flavors become active at $1 \mathrm{TeV}$ we find that $g_{s}=4 \pi$ for $\mu \sim 2000 \mathrm{TeV}$ where the instanton vertex will become unsuppressed.

We will assume that some UV degrees of freedom cut off this growth of this coupling above a scale $M \sim 2000 \mathrm{TeV}$ such that $\kappa_{s}$ has a maximal value at this scale. We will treat this maximal value as a free parameter that can vary from $\kappa_{s}=10^{-34}$ for $g_{s}=1$ to $\kappa_{s} \sim 1$ for $g_{s}=4 \pi$. We will also assume that at a scale $M^{\prime}>M$, the QCD gauge coupling growth is tamed, $\kappa_{s}$ becomes negligible and the QCD instantons are again highly suppressed.

\section{B. Running of $\lambda_{f}^{i j}$}

We will work in the mass basis where $\lambda_{u L}^{i j}\left(M^{\prime}\right)=$ $\operatorname{diag}\left[y_{u L}, y_{c L}, y_{t L}\right]$ and $\lambda_{d L}^{i j}\left(M^{\prime}\right)=\operatorname{diag}\left[y_{d L}, y_{s L}, y_{b L}\right]$. The couplings $y_{f}$ run between the UV and IR scale, $m_{\star} \sim 1 \mathrm{TeV}$, of the composite masses,

$$
\mu \frac{d y_{f}}{d \mu}=\left(d_{F}-5 / 2\right) y_{f}+b \frac{N_{\mathrm{HC}} y_{f}^{3}}{16 \pi^{2}}
$$

where $b$ is an $\mathcal{O}(1)$ factor, $N_{\mathrm{HC}}=4$ is the number of colors for the hypercolor group and $d_{F}$ is the conformal dimension of the operator corresponding to the fermionic partner, $F$, that couples to the SM fermion, $f$. The first term allows an anarchic and $\mathcal{O}(1)$ valued matrix $\lambda_{f}^{i j}(M)$ to generate a hierarchical $\lambda_{f}^{i j}\left(m_{\star}\right)$ thus explaining the SM masses and mixings. This can be seen if we solve the above equation by ignoring the second term,

$$
y_{f}\left(m_{\star}\right)=y_{f}(M)\left(\frac{m_{\star}}{M}\right)^{d_{F}-5 / 2},
$$

which shows that $\mathcal{O}(1)$ differences in the $d_{j}$ can lead to exponential hierarchies in the IR. Including the second term does not change this qualitative feature, in fact it can lead to hierarchies between couplings involving operators with the same $d_{F}$. In particular it results in a fixed point at $y_{f}=$ $4 \pi / \sqrt{b N_{\mathrm{HC}} \gamma_{F}}$ where $\gamma_{F}=d_{F}-5 / 2$.

\section{UP QUARK MASS FROM SMALL INSTANTONS}

In this section we will consider the model defined in Sec. II and assume that one of the eigenvalues of $\lambda_{u L}^{i j}$ vanishes at the scale $M^{\prime}$ where instanton effects are negligible, i.e., $y_{u L}\left(M^{\prime}\right)=0$. Instanton effects around the scale $M$ then generate a nonzero value for the up quark mixing, $y_{u L}$, via the 't Hooft vertex. We will work with the lagrangian obtained after all the phase redefinitions in Sec. II E have been performed.

\section{A. Nonperturbative generation of $y_{u L}$}

The 't Hooft instanton vertex due to the QCD anomaly in this model is an interaction including all the colored fermion species. In Fig. 2(a) we show only the first generation fermions, $u_{L}, u_{R}^{c}, d_{L}, d_{R}^{c}, \chi_{u}^{1}, \tilde{\chi}_{u}^{1}, \chi_{d}^{1}$ and $\tilde{\chi}_{d}^{1}$ explicitly. Working in the mass basis we start from the anomaly vertex to generate the $y_{u L} u_{L} U_{L}^{c 1}$ term as shown in Fig. 2(a). The fermions of the other generations have not been shown for space constraints but a identical topology exists for them with the only difference that now the $c_{L}-U_{L}^{c 2}$ and $t_{L}-U_{L}^{c 3}$ lines are also closed by the couplings $y_{c L}^{*}$ and $y_{t L}^{*}$.

To get the NDA estimate for $y_{u L}$ we can redraw the same diagram but now in terms of QCD and hypercolor singlets as shown in Fig. 2(b). If one considers the pairs, $U_{L}^{c 1} U_{R}^{1}$, $U_{L}^{c 1} U_{L 0}^{c 1}, D_{L}^{c 1} D_{R}^{1}$, and $D_{L}^{c 1} D_{L 0}^{c 1}$ as QCD singlet scalars, this diagram becomes very similar to the one considered in Ref. [11] where new scalars connect fermion pairs, such as $u_{L} u_{R}^{c}$ to $d_{L} d_{R}^{c}$, in the 't Hooft vertex. The result of a full calculation in Ref. [11] is that the only suppression factor is given by, $\prod_{f} \mathcal{Y}_{f} / 4 \pi$, where the $\mathcal{Y}_{f}$ are the Yukawa couplings of the scalars to the fermion pairs. In our case the coupling of the SM fermions to the scalars, formed from the composite partners, reaches its perturbative limit for $y_{f L}^{*}=y_{f R}^{*}=4 \pi$. Thus adapting the result of Ref. [11] to our case, and including a suppression factor corresponding to $g_{4 \psi}$, we obtain,

$$
\frac{y_{u L}}{4 \pi} \sim \kappa_{s}\left(\frac{g_{4 \psi}}{16 \pi^{2}}\right)^{3} \frac{y_{u R}^{*}}{4 \pi} \prod_{f=d, s, c, b, t} \frac{y_{f_{L}}^{*}}{4 \pi} \frac{y_{f_{R}}^{*}}{4 \pi},
$$

where all the above couplings are at the scale $M$, and following Eq. (9), all the $y_{f R}=y_{u R, d R}$ depending on whether $f$ is an up or down type fermion. The white circles in Fig. 2(b) represent vertices arising from the strong sector whereas the dark circles denote vertices external to the strong sector. Here we have assumed a suppression only due to the former couplings.

The known value of the up quark Yukawa can be reproduced in the strongly coupled regime when the couplings in Eq. (16) saturate their perturbative limit. For instance we obtain for the up Yukawa,

$$
Y_{u}\left(m_{\star}\right) \sim \frac{y_{u L}\left(m_{\star}\right) y_{u R}\left(m_{\star}\right)}{4 \pi} \sim 1.5 \times 10^{-5} \kappa_{s}\left(\frac{g_{4 \psi}}{16 \pi^{2}}\right)^{3}
$$

if we solve the RG equations in Eq. (14) assuming $b=1 / 4$, taking $d_{U_{L}, D_{L}}=7 / 2, \quad y_{u R, d R}(M)=4 \pi \quad$ in Eq. (9), $y_{u R, d R}\left(m_{\star}\right)=y_{u R, d R}(M) / 10, \quad$ and other boundary 


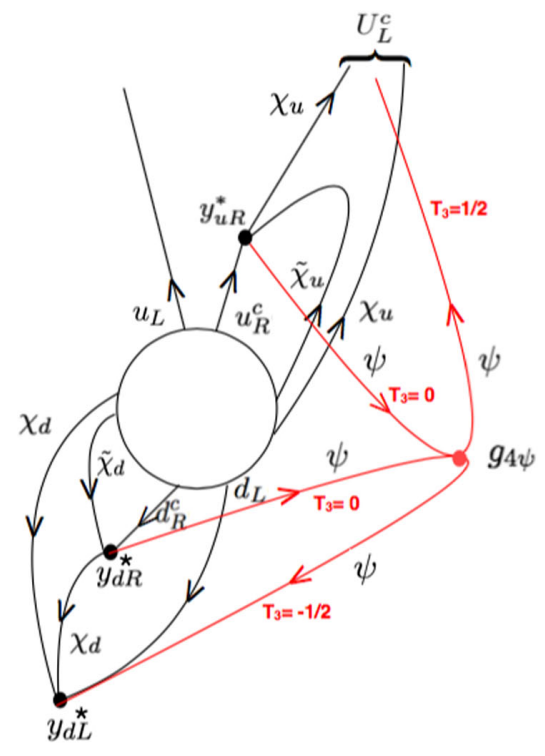

(a)

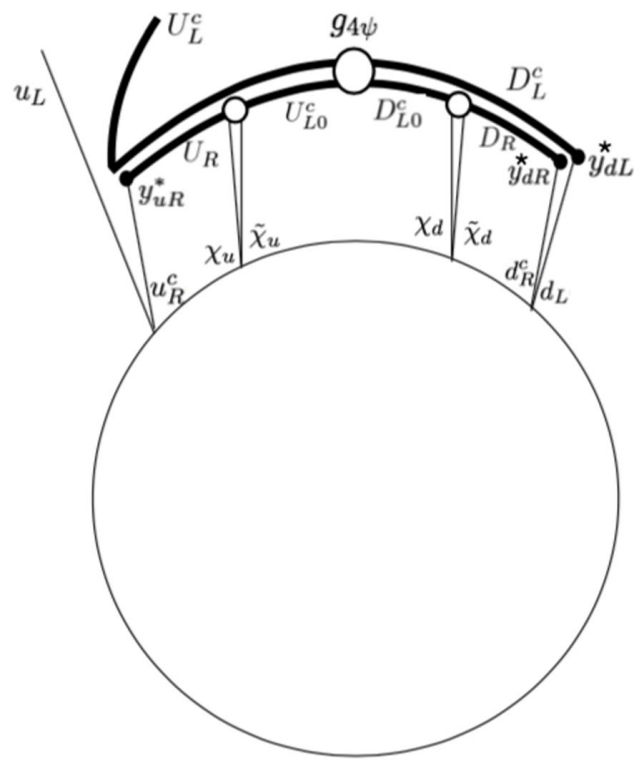

(b)

FIG. 2. (a) Diagram showing the QCD instanton contribution to generate $u_{L} U_{L}^{c}$ including only the first generation of fermions; the generation indices for the hypercolor fermions have been omitted for convenience. An identical topology exists for the other generations with the only difference that the $c_{L}-U_{L}^{c 2}$ and $t_{L}-U_{L}^{c 3}$ lines are closed by the couplings $y_{c L}^{*}$ and $y_{t L}^{*}$. The red lines do not intersect with the others. (b) The same diagram drawn in terms of lines of hypercolor and QCD singlets. The white circles represent unsuppressed vertices arising from the strong sector whereas the dark circles denote vertices suppressed by couplings external to the strong sector.

conditions fixed by the measured value of the SM fermions masses.

\section{B. Solution to the strong $C P$ problem}

Let us first consider the scale $M^{\prime}$ at and above which QCD instanton effects are suppressed so that $y_{u L}\left(M^{\prime}\right)=0$. At this scale the phase $\theta_{\mathrm{QCD}}$ can be removed simply by a chiral rotation of the up quark fields.

It is still instructive to see how, at leading order, $\theta_{\mathrm{QCD}}$ vanishes just below the scale $M$ where $\mathrm{QCD}$ instanton effects become important. These effects generate a nonzero $y_{u L}$ given by Eq. (16). As explained in Sec. II E, we are working in a convention where chiral rotations are used to transfer all the phases to the mixing matrices $\Lambda_{\not /}^{i j}$ and there is no $G \tilde{G}$ coupling to start with. A contribution to $\bar{\theta}_{\mathrm{QCD}}$ from the fermionic phases can arise at this scale from closing the 't Hooft vertex completely, which gives,

$$
\begin{aligned}
\bar{\theta}_{\mathrm{QCD}}(M) & =\operatorname{Arg}\left(y_{u L}^{*} y_{u R}^{*} \prod_{f=d, s, c, b, t} y_{f L}^{*} y_{f R}^{*}\right) \\
& =\operatorname{Arg}\left(\prod_{f=u, d, s, c, b, t}\left|y_{f L}\right|^{2}\left|y_{f R}\right|^{2}\right)=0 .
\end{aligned}
$$

where we used Eq. (16) for deriving the second line. While Eq. (16) gives the leading contribution to $y_{u L}$, subleading contributions from other diagrams that close the 't Hooft vertex are possible. These contributions, however, will have the same parametric dependance as Eq. (16) because of the fact that our set-up realizes MFV as shown in Sec. II E. Furthermore, the fact that all phases in our Lagrangian can be transferred to the $\lambda_{u L, d L}^{i j}$ means that there will be no relative phase between the different contributions. Their final contribution to $\bar{\theta}_{\mathrm{QCD}}(M)$ will thus vanish as in the equation above.

In Appendix we discuss whether higher dimensional operators that break the global symmetries in our model, in particular those that lead to a violation of MFV, can spoil the success of our mechanism. We find that not only is our framework robust when Planck scale global symmetry breaking effects are included, it can be successful even if we allow the breaking of global symmetries at the scale $\Lambda \sim M$.

There is only one more physical phase in our theory, the CKM phase, $\theta_{\mathrm{CKM}}$, as explained in Sec. II E. From the scale $M$ to the experimental scale, $\theta_{\mathrm{CKM}}$ can induce $\bar{\theta}_{\mathrm{QCD}}$ due to renormalization group (RG) effects, but as in the SM this is expected to be highly suppressed. This is because the arguments based on spurion analysis for the SM in Ref. [23] can be adapted to our model to show that this effect is at least 7-loop suppressed [24].

\section{A HEAVY AXION FROM SMALL INSTANTONS}

Now we consider a different scenario from Sec. IV, taking $y_{u L}\left(M^{\prime}\right) \neq 0$, but introduce a new pseudoscalar field, 
$\phi$. In addition to all the Lagrangian terms in Sec. II, we consider the new term,

$$
\mathcal{L}_{\phi}=\frac{g_{s}^{2}}{32 \pi^{2}} \frac{\phi}{f} G_{\mu \nu} \tilde{G}^{\mu \nu}
$$

where we take $f \gtrsim M \sim 10^{6} \mathrm{GeV}$. We assume that above term is the only one that breaks the shift symmetry of $\phi$ via anomalous QCD effects so that it can be identified with the QCD axion.

Nonperturbative effects at the scale $M$ in the UV as well as the usual QCD scale in the IR will contribute to the axion potential. The UV contribution can be estimated by first shifting $\bar{\theta}_{\mathrm{QCD}}$ to the 't Hooft vertex in Fig. 2(a) and 2(b) and then completely closing all the fermion lines which is possible now that $y_{u L} \neq 0$. This then gives a new contribution to the axion potential,

$$
V(\phi)=\kappa M^{4} \cos \left(\frac{\phi}{f}+\bar{\theta}_{\mathrm{QCD}}\right)+m_{\pi}^{2} f_{\pi}^{2} \cos \left(\frac{\phi}{f}+\bar{\theta}_{\mathrm{QCD}}\right)
$$

where,

$$
\kappa \sim \kappa_{s}\left(\frac{g_{4 \psi}}{16 \pi^{2}}\right)^{3} \prod_{f=u, d, s, c, b, t} \frac{y_{f L}^{*}}{4 \pi} \frac{y_{f R}^{*}}{4 \pi} .
$$

Again, all the above couplings are at the scale $M$ and all the $y_{f R}=y_{u R, d R}$ depending on whether $f$ is an up or down type fermion [see Eq. (9)]. The second term above is the usual large instanton contribution in the IR, with $m_{\pi}$ being the pion mass and $f_{\pi}$, the pion decay constant. Both the terms are aligned in phase because both the contributions arise from closing the same 't Hooft vertex, but at different scales. As in Sec. IV-the fact that MFV is respected in our set-up and that all phases can be shifted to $\lambda_{u L, d L}^{i j}$, means that any subleading part of the small instanton contribution to the axion potential at the scale $M$, must be aligned in phase to the terms in Eq. (20). We show in Appendix that higher dimensional operators that break MFV can be rendered harmless under some plausible assumptions.

Solving the RG equations in Eq. (14) assuming $b=1 / 4$, taking $d_{U_{L}, D_{L}}=7 / 2, y_{u R, d R}(M)=4 \pi$ in Eq. (9), $y_{u R, d R}\left(m_{\star}\right)=y_{u R, d R}(M) / 10$, and other boundary conditions fixed by the measured value of the SM fermions masses, we obtain,

$$
\kappa \sim \kappa_{s}\left(\frac{g_{4 \psi}}{16 \pi^{2}}\right)^{3} 4.2 \times 10^{-4} .
$$

The above numerical value is the maximal possible one corresponding to the case when all the couplings saturate their perturbative limit. The factor, $\kappa_{s}$, can vary over a large range from unity to exponentially small values as the strong coupling is varied; the axion mass can thus vary

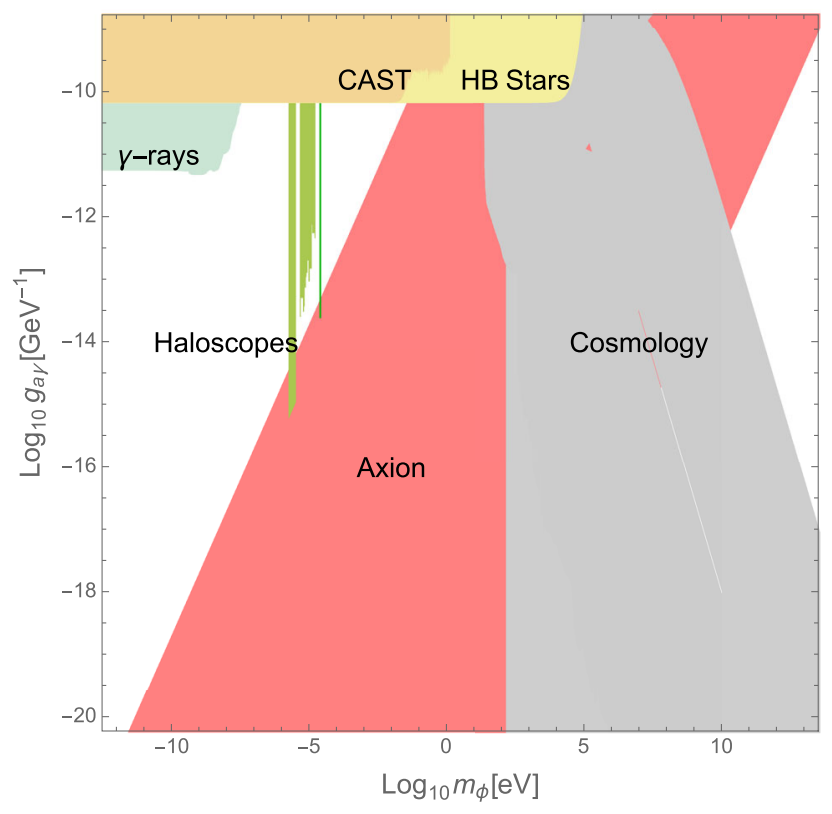

FIG. 3. The allowed parameter space for the QCD axion of Sec. V is shown in red. The other bounds have been adapted from Refs. [14,27] where a detailed discussion of these can be found.

from its minimum value due to the IR contribution to values as large as $10 \mathrm{TeV}$ when the suppression factor, $\kappa$ approaches the maximal value in Eq. (21).

We show the allowed region in the coupling-mass parameter space in Fig. 3, where the $y$-axis is $g_{a \gamma}$ the axion coupling to photons, defined by the coupling,

$$
-\frac{g_{a \gamma}}{4} \phi F_{\mu \nu} \tilde{F}^{\mu \nu}
$$

where,

$$
g_{a \gamma} \sim \frac{\alpha_{e m}}{2 \pi f} .
$$

We see that a huge area in the parameter space is allowed for the QCD axion in this model. The usual QCD axion band is the left edge of the area shown in Fig. 3. While parts of this area are ruled out by existing constraints-in particular cosmological ones in the region where a thermal population of the axion can exist [27]—-large parts of the allowed region still remain unconstrained.

\section{CONCLUSIONS}

In this work we showed that small instanton effects can become very important in standard UV completions of composite Higgs models with partially composite fermions. This is possible because both the QCD gauge coupling and effective Yukawa interactions run to larger values with energy resulting in unsuppressed instanton contributions in the UV. As far as the Yukawa interactions are concerned, it is well known that in partially composite 
models, the hierarchical nature of the SM fermion masses and mixings can arise from anarchic and $\mathcal{O}(1)$ interactions in the UV. The QCD coupling grows because in order to have fermionic partners for all SM fermions, many new fermions in the hypercolor sector need to be introduced. These fermions are also charged under QCD and this generates a positive $\beta$-function which results in the $\mathrm{QCD}$ gauge coupling running to nonperturbative values in the UV. The only modification of the lagrangian required to achieve this effect are the explicit breaking terms in Eq. (7).

As a consequence of this enhancement of small instanton contributions, we show that the up quark mass can arise entirely from instanton contributions. This implies that the up quark mass vanishes in the deep UV and is only generated additively by instanton effects. In the deep $\mathrm{UV}$ one can thus rotate away the strong $C P$ phase. In an alternative scenario where an axion field exists, we show that its mass can be as large as $10 \mathrm{TeV}$. The allowed parameter space is much larger than the usual QCD band as shown in Fig. 3. Our model thus opens up new areas in the coupling-mass parameter space that are still unconstrained by existing bounds. This motivates the development of new experimental strategies to probe these regions.

\section{ACKNOWLEDGMENTS}

We would like to thank A. Pomarol for his feedback on this work and for some key suggestions on the model. R. S. G. would like to thank C. Csaki for encouraging him to think about the 't Hooft anomalies of the model; this led to the identification of some presentation errors in the first version of this manuscript which have now been corrected.

Note added.-While we were in the process of completing this project, Ref. [28] appeared, that also provides a heavy axion candidate in composite models. While this work also utilizes the setup of Ref. [19], there is no overlap with Sec. V. This is because Ref. [28] does not use the same mechanisms for enhancing small instanton contributions, namely running of the QCD gauge coupling and the $\lambda_{f}^{i j}$, that have been used in this work. The other important differences include the fact that Ref. [28] has an in-built axion candidate and, unlike this work, it requires a hypercolor condensation scale of at least $1000 \mathrm{TeV}$ resulting in a tuned Higgs sector.

\section{APPENDIX: EFFECT OF HIGHER DIMENSIONAL OPERATORS}

In this appendix we discuss whether higher dimensional operators can spoil our solution to the strong $C P$ problem. Our analysis so far has been based on the assumption that the only couplings breaking the $S U(3)_{F}^{u} \times S U(3)_{F}^{d}$ flavor symmetry are the left-handed mixings, $\lambda_{u L, d L}^{i j}$, to which all other phases in our lagrangian can be transferred as explained in Sec. IIE. Under this assumption our setup respects minimal flavor violation (MFV). As explained below Eq. (16) and Eq. (20) this ensures that there are no subleading contributions, misaligned in phase to the up mass in Sec. IV or to the heavy axion potential in Sec. V.

We now discuss to what extent our mechanism is robust against the effect of higher dimensional operators that introduce a new breaking of this flavor symmetry. For instance any new contribution of these operators to the mass matrix of SM fermions will break our MFV assumption. The most dangerous effects can arise from operators with two flavor indices, such as,

$$
\bar{Q}_{L}^{i} \mathcal{O}_{H} u_{R}^{j}
$$

which introduces a SM like 'Yukawa' coupling that couples the Higgs operator, $\mathcal{O}_{H}=\psi_{-+} \psi_{0}$, to SM fermions, or operators like,

$$
u_{R}^{c, i}\left(\overline{\tilde{\chi}}_{u} \psi \overline{\tilde{\chi}}_{u}\right)^{j}, \quad u_{R}^{c, i}\left(\chi_{u} \psi \chi_{u}\right)^{j}
$$

which correspond to bilinear mixings of SM fermions with operators other than those in Eq. (6), and finally the mass term,

$$
m_{\chi u}^{i j} \chi_{u}^{i} \tilde{\chi}_{u}^{j}
$$

For simplicity, we do not explicitly discuss the corresponding operators with the SM quark doublets or the down-type singlets which can be easily included along the same lines while keeping our conclusions unchanged. All these operators are prohibited if we impose a discrete $Z_{2}$ gauge symmetry, already introduced in Sec. II C, under which under which $\tilde{\chi}_{u, d}^{i}$ and the SM right chiral fermions are odd.

Operators with even higher dimensions, that respect this $Z_{2}$ symmetry can, however, still give dangerous contribution to the fermion masses,

$$
\begin{array}{cc}
\frac{1}{\Lambda_{\not p}^{d 1}} \tilde{\chi}_{u}^{i} \chi_{u}^{i} \bar{Q}_{L}^{i} \mathcal{O}_{H} u_{R}^{j} & \frac{1}{\Lambda_{\not p}^{d 2}} u_{R}^{c, i} \tilde{\chi}_{u}^{i} \chi_{u}^{i}\left(\overline{\tilde{\chi}}_{u} \psi \overline{\tilde{\chi}}_{u}\right)^{j} \\
\frac{1}{\Lambda_{\not p}^{d 3}} u_{R}^{c, i} \tilde{\chi}_{u}^{i} \chi_{u}^{i}\left(\chi_{u} \psi \chi_{u}\right)^{j} & \frac{1}{\Lambda_{\not f}^{d 4}} \tilde{\chi}_{u}^{i} \chi_{u}^{i} \chi_{u}^{i} \tilde{\chi}_{u}^{j}
\end{array}
$$

where $d_{1}, d_{2}, d_{3}$, and $d_{4}$ are the scaling dimensions of the respective operators. Note that these operators break, not only the $S U(3)_{F}^{u} \times S U(3)_{F}^{d}$ flavor symmetry, but also the $S U(5)$ global symmetry in the electroweak sector because the SM quarks are not in full $S U(5)$ representations. The contributions of these operators are, however, suppressed by the factor,

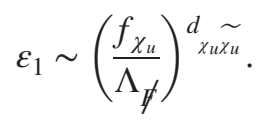


for the first three operators and by the factor,

$$
\varepsilon_{2} \sim\left(\frac{f_{\chi_{u}}}{\Lambda_{\not f}}\right)^{2 d_{\chi_{u} \chi_{u}}}{ }^{-4},
$$

for the last one. Recall that $d_{\chi_{u} \tilde{\chi}_{u}}$ is the scaling dimension of the condensate, $\left\langle\tilde{\chi}_{u}^{i} \chi_{u}^{i}\right\rangle$.

For the case in Sec. IV where we generate the mass of the up quark from small instantons, the above dangerous operators can spoil our strong $C P$ solution by either generating a mixing of the left handed up to the strong sector, i.e., $y_{u L} \neq 0$ at scales larger than $M$, or by generating a small instanton contribution misaligned in phase to the one in Eq. (16). Both these contributions can be bounded by requiring that the resulting $\bar{\theta}_{\mathrm{QCD}}$ is within the experimental bound, $\bar{\theta}_{\mathrm{QCD}} \lesssim 10^{-10}$, which implies,

$$
\varepsilon_{1,2} \lesssim 10^{-10}
$$

The above conditions will also ensure that any misaligned contribution to the potential of the heavy axion in Sec. V is within the current bound on $\bar{\theta}_{\mathrm{QCD}}$.

If MFV and the $S U(5)$ global symmetry is broken only by Planck scale operators, i.e. if we take $\Lambda_{\not p}=M_{p l}$, the conditions in Eq. (A7) is easily satisfied given $f_{\chi_{u}}=1 \mathrm{TeV}$. Next we consider the case where the flavor symmetry is broken at the scale, $\Lambda_{\not f}=\Lambda=M=2000 \mathrm{TeV}$, by the states required to generate the bilinear mixing terms in Eq. (6) or by those required to tame the running of the QCD coupling at the scale $M$. We see that for any $d_{\chi_{u} \widetilde{\chi_{u}}} \geq$ $7 / 2$ the conditions in Eq. (A7) can be satisfied even in this case.

Thus, our setup is completely robust if the global symmetries are broken by only Planck-scale effects. Even if we allow higher dimensional explicit breaking effects at the scale $\Lambda_{\not f}=\Lambda=M$, we find that our mechanism is safe under some plausible assumptions.
[1] R. D. Peccei and H. R. Quinn, $C P$ Conservation in the Presence of Instantons, Phys. Rev. Lett. 38, 1440 (1977).

[2] S. Weinberg, A New Light Boson? Phys. Rev. Lett. 40, 223 (1978).

[3] F. Wilczek, Problem of Strong $P$ and $T$ Invariance in the Presence of Instantons, Phys. Rev. Lett. 40, 279 (1978).

[4] H. Georgi and I. N. McArthur, Instantons and the mu quark mass, Harvard perprint (1981) HUTP-81/A011.

[5] K. Choi, C. W. Kim, and W. K. Sze, Mass Renormalization by Instantons and the Strong CP Problem, Phys. Rev. Lett. 61, 794 (1988).

[6] D. B. Kaplan and A. V. Manohar, Current Mass Ratios of the Light Quarks, Phys. Rev. Lett. 56, 2004 (1986).

[7] T. Banks, Y. Nir, and N. Seiberg, Missing (up) mass, accidental anomalous symmetries, and the strong $C P$ problem, arXiv:hep-ph/9403203.

[8] M. Dine, P. Draper, and G. Festuccia, Instanton effects in three flavor QCD, Phys. Rev. D 92, 054004 (2015).

[9] S. Aoki et al., Review of lattice results concerning lowenergy particle physics, Eur. Phys. J. C 77, 112 (2017).

[10] C. Alexandrou et al., Ruling Out the Massless Up-Quark Solution to the Strong $\boldsymbol{C P}$ Problem by Computing the Topological Mass Contribution with Lattice QCD, Phys. Rev. Lett. 125, 232001 (2020).

[11] J. M. Flynn and L. Randall, A computation of the small instanton contribution to the axion potential, Nucl. Phys. B293, 731 (1987).

[12] B. Holdom and M. E. Peskin, Raising the axion mass, Nucl. Phys. B208, 397 (1982).

[13] T. Gherghetta, V. V. Khoze, A. Pomarol, and Y. Shirman, The axion mass from 5D small instantons, J. High Energ. Phys. 03 (2020) 063.
[14] P. Agrawal and K. Howe, Factoring the strong $C P$ problem, J. High Energy Phys. 12 (2018) 029.

[15] P. Agrawal and K. Howe, A flavorful factoring of the strong CP problem, J. High Energy Phys. 12 (2018) 035.

[16] C. Csaki, M. Ruhdorfer, and Y. Shirman, UV sensitivity of the axion mass from instantons in partially broken gauge groups, J. High Energy Phys. 04 (2020) 031.

[17] K. Agashe, R. Contino, and A. Pomarol, The minimal composite Higgs model, Nucl. Phys. B719, 165 (2005).

[18] G. Panico and A. Wulzer, The Composite Nambu-Goldstone Higgs (Springer, New York, 2016), Vol. 913.

[19] G. Ferretti, UV completions of partial compositeness: The case for a SU(4) gauge group, J. High Energy Phys. 06 (2014) 142.

[20] B. Holdom, Raising the Sideways Scale, Phys. Rev. D 24, 1441 (1981).

[21] M. Redi and A. Weiler, Flavor and $C P$ invariant composite Higgs models, J. High Energy Phys. 11 (2011) 108.

[22] G. D’Ambrosio, G. Giudice, G. Isidori, and A. Strumia, Minimal flavor violation: An effective field theory approach, Nucl. Phys. B645, 155 (2002).

[23] J. R. Ellis and M. K. Gaillard, Strong and weak $C P$ violation, Nucl. Phys. B150, 141 (1979).

[24] The diagrams shown in Ref. [23] for the SM would apply for our model as long as the Higgs fermion couplings in the SM diagram are appropriately replaced by strong dynamics. Apart from RG effects there are also finite contributions to the strong $C P$ phase from the CKM phase, but in Ref. [23] these were also shown to be much smaller than the experimental bound, $\bar{\theta}_{\mathrm{QCD}} \lesssim 10^{-10}$, from neutral electric dipole moment experiments $[25,26]$. 
[25] M. Pospelov and A. Ritz, Theta Induced Electric Dipole Moment of the Neutron via QCD Sum Rules, Phys. Rev. Lett. 83, 2526 (1999).

[26] C. Baker et al., An Improved Experimental Limit on the Electric Dipole Moment of the Neutron, Phys. Rev. Lett. 97, 131801 (2006).
[27] D. Cadamuro and J. Redondo, Cosmological bounds on pseudo Nambu-Goldstone bosons, J. Cosmol. Astropart. Phys. 02 (2012) 032.

[28] T. Gherghetta and M. D. Nguyen, A composite Higgs with a heavy composite axion, J. High Energy Phys. 12 (2020) 094. 